\title{
Improved Lesion Detection Using Nonlocal Means Post-Processing
}

\author{
Ole Marius Hoel Rindal*, Alfonso Rodriguez-Molares* ${ }^{* \dagger}$, Svein-Erik Måsøy ${ }^{\dagger \dagger}$ and Tore Grüner Bjåstad ${ }^{\dagger \ddagger}$ \\ * Department of Informatics \\ University of Oslo \\ Oslo, Norway \\ Email: omrindal@ifi.uio.no \\ $\dagger^{\dagger}$ Department of Circulation and \\ Medical Imaging \\ Norwegian University of Science and \\ Technology \\ Trondheim, Norway \\ ‡InPhase Solutions AS \\ Trondheim, Norway
}

\begin{abstract}
Software beamforming allows more flexible and complex algorithms, often referred to as adaptive beamforming techniques, that are blurring the boundaries between beamforming and image processing. Many adaptive beamforming algorithms claim to improve lesion detectability. Based on recent advances, we hypothesize that image processing techniques that reduce speckle variability yield better lesion detectability than state-of-the-art adaptive beamformers.

This hypothesis is investigated on six algorithms: two image processing techniques, and four adaptive beamformers. As a target we use Field II simulations of a hypoechoic cyst with noise added to simulate different SNR conditions. Lesion detectability is estimated using the Generalized Contrast-to-Noise Ratio (GCNR). The results support our hypothesis.

Index Terms-Lesion detection, adaptive beamforming, coherence beamforming, Generalized Contrast-to-Noise Ratio.
\end{abstract}

\section{INTRODUCTION}

Lesion detectability of an imaging method has traditionally been estimated using either the contrast ratio (CR) or the contrast-to-noise ratio (CNR). It has recently been shown that $\mathrm{CR}$ and $\mathrm{CNR}$ can be arbitrarily increased by dynamic range transformations [1]. A new metric has been introduced, the generalized-contrast-to-noise ratio (GCNR) [2], which estimates the maximum classification rate that can be achieved by an optimal observer.

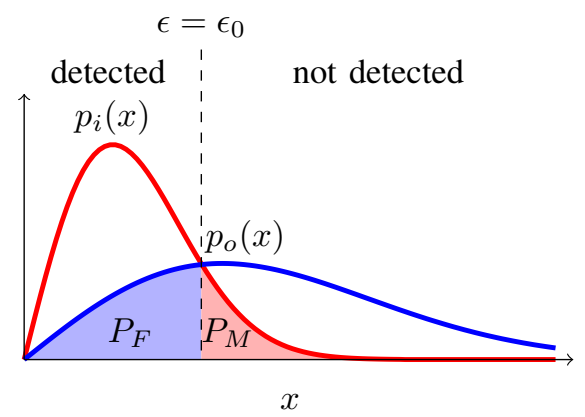

Fig. 1: Illustration of the two probability density functions $p_{i}(x)$ and $p_{o}(x)$, and the miss-classification probabilities $P_{F}(\epsilon)$ and $P_{M}(\epsilon)$ for an optimal threshold $\epsilon_{0}$.
Using this metric, it becomes apparent that coherencebased beamforming algorithms are not particularly good at increasing the detection of uniform cysts, those typically used to study lesion detectability. Other post-processing techniques, aimed to reduce the speckle variance, could outperform these adaptive beamformers at this particular task. We aim to test this hypothesis.

This paper is structured as follows. Section II describes the GCNR metric. Section III presents the tested beamformers and the image processing techniques. Section IV presents the results, that are discussed in Section V. Some concluding remarks are included in Section VI.

\section{BACKGROUND}

1) The Generalized Contrast-to-Noise Ratio (GCNR): was introduced in [2] as a measure of lesion detectability. It was shown that GCNR has the following properties:

1) it is resistant to dynamic range alterations;

2) it can be used on any kind of data, regardless of the signal nature or units; and

3 ) it is a quantitative metric with physical meaning: the amount of pixels that are correctly classified by an optimal observer.

The GCNR is calculated as

$$
\mathrm{GCNR}=1-\mathrm{OVL}
$$

where OVL is the overlapping region between the probability density functions (PDFs) for the pixels inside $p_{i}(x)$ and outside $p_{o}(x)$ the lesion. This overlapping region consists of the rate of pixels that have been falsely detected outside the lesion, $P_{F}$; plus the rate of those that have been missed inside the lesion, $P_{M}$. An illustration of $p_{i}(x)$ and $p_{o}(x)$ is shown in Fig. 1 for an optimal detection treshold $\epsilon_{0}$, and the resulting OVL indicated by the two colored regions.

Under certain circumstances, an analytical expression for the GCNR of conventional delay-and-sum can be derived [2]

$$
\mathrm{GCNR}_{0}=\mathrm{C}_{0}^{-\frac{\mathrm{C}_{0}}{\mathrm{C}_{0}-1}}-\mathrm{C}_{0}^{-\frac{1}{\mathrm{C}_{0}-1}} \text {. }
$$


where $\mathrm{C}_{0}$ can be calculated dependant on the number of elements $M$ and the channel SNR

$$
\mathrm{C}_{0}=\frac{3}{2 M \mathrm{SNR}+3} \text {. }
$$

\section{Methods}

We used Field II [3][4] to simulate a $6 \mathrm{~mm}$ diameter anechoic cyst using a synthetic transmit aperture sequence and a 128 -element, $300 \mathrm{um}$, linear probe transmitting at $5.13 \mathrm{MHz}$. A total of 20 datasets were generated. Band-pass Gaussian noise was added with different intensities to simulate channel SNR conditions from -20.7 to $4.4 \mathrm{~dB}$.

The datasets were processed using 6 image formation methods:

1) delay-and-sum and spatial averaging (DAS + SA), using a 2D kernel of 30 pixels;

2) delay-and-sum and nonlocal means [5] (DAS + NLM), with parameters resulting in a search radi and comparison radi of 30 by 30 pixels, a preselection threshold of $4, \sigma=80$ assuming a Gaussian distribution of the pixels;

3) phase coherence factor (PCF) [6], with $\gamma=1$;

4) generalized coherence factor (GCF) [7], with $M_{0}=4$;

5) short lag spatial coherence (SLSC- $\lambda$ ) [8], with $M_{\max }=$ 14 and a $\lambda$ kernel size; and

6) short lag spatial coherence (SLSC-0.1 $\lambda$ ) [8], with $M_{\max }=14$ and a $0.1 \lambda$ kernel size.

Both spatial averaging and nonlocal means was applied after envelope detection and logarithmic compression. GCNR was estimated using Eq. (1) for all SNR conditions. Fig. 4a shows the regions $i$, red, and $o$, blue, used to calculate GCNR.

All processing was done in MATLAB (The MathWorks, Natick, USA) using the UltraSound ToolBox (USTB) [9]. The data and code needed to produce all figures and results are available at http://www.ustb.no.

\section{Results}

Fig. 3 validates the analytical expression for the GCNR of DAS, Eq. (2), against the Field II simulation. Snapshots of the DAS images and the probability density functions $p_{i}(x)$ and $p_{o}(x)$ are shown in Fig. 4 for a channel SNR of $-4.86 \mathrm{~dB}$ and $-14.07 \mathrm{~dB}$. As expected better separation of both PDFs is observed in high SNR conditions, leading to higher GCNR values.

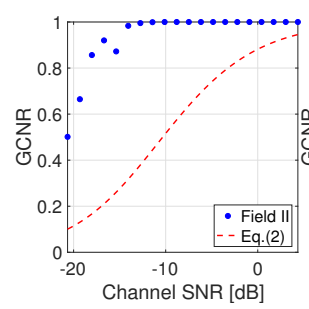

(a) DAS + SA

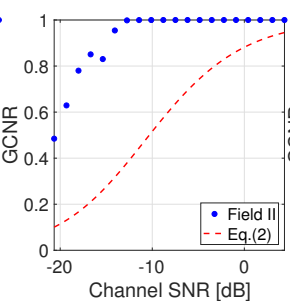

(b) DAS + NLM

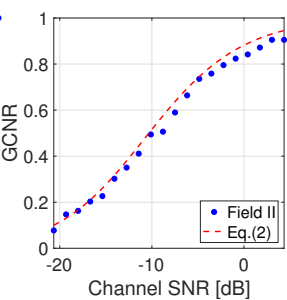

(c) $\mathrm{PCF}$

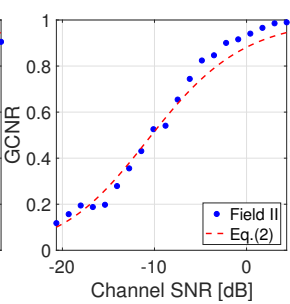

(d) GCF

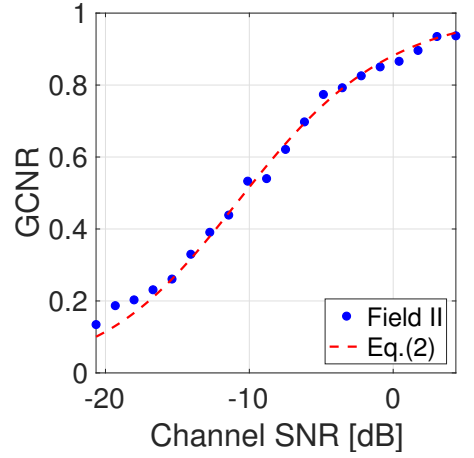

Fig. 3: GCNR values for DAS vs Eq. (2)

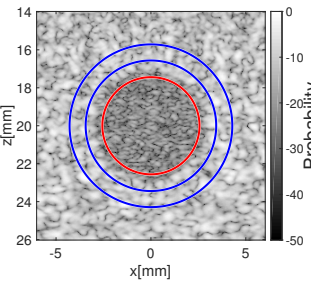

(a) DAS, SNR=-4.85 dB

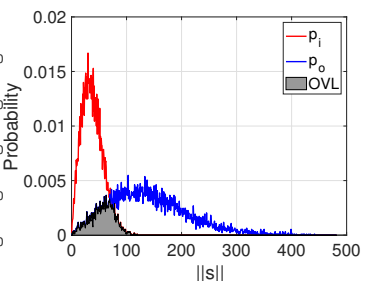

(b) DAS, SNR $=-4.85 \mathrm{~dB}$

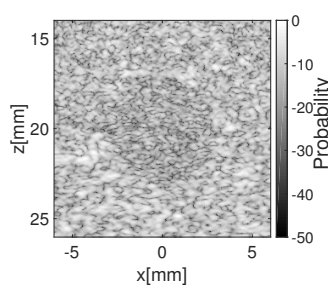

(c) DAS, SNR $=-14.07 \mathrm{~dB}$

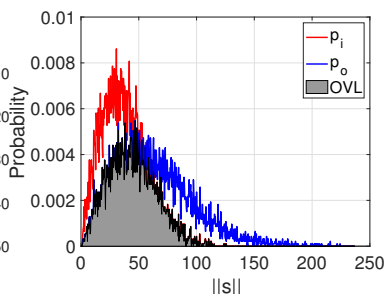

(d) DAS, SNR=-14.07 dB
Fig. 4: Snapshots of the DAS images and $p_{i}(x)$ and $p_{o}(x)$ for a channel SNR of $-4.86 \mathrm{~dB}$ (top row) and $-14.07 \mathrm{~dB}$ (bottom row).

Fig. 2 shows the GCNR values vs SNR for the 6 tested methods. We observe that the post-processing methods outperform the adaptive beamformers for all SNR conditions. Trivial DAS + SA, and DAS + NLM show very similar lesion detectability. PCF, GCF, and SLSC-0.1 $\lambda$ are also very similar, with almost negligible variations from DAS. SLCS- $\lambda$ shows a significant improvement in lesion detection over DAS, but still far smaller than that of the post-processing approaches.

Fig. 2: GCNR values obtained for the six tested methods. The red dashed line in the GCNR plot is the analytical derivation the GCNR of DAS, Eq. (2), while the blue dots are the estimated GCNR for the SNR level indicated on the x-axis. 
For completeness, we include in Fig. 6 the snapshots of the resulting images and probability density functions $p_{i}(x)$ and $p_{o}(x)$ for a channel SNR of $-4.86 \mathrm{~dB}$ and $-14.07 \mathrm{~dB}$.

\section{DiscusSiON}

Our results show that DAS $+\mathrm{SA}$ and DAS + NLM provide better lesion detectability than the tested coherence methods. This makes good sense: these averaging techniques reduce speckle variance both within and outside the lesion and make the separation of both regions easier. This is confirmed by the plots in Fig. 6, where we observe that, both, DAS + SA and DAS + NLM, reduce the variance of $p_{i}$ and $p_{o}$ while increasing the distance between their means.

High lesion detectability can be achieved by trivial spatial averaging (i.e. DAS + SA), however DAS + NLM does a better job at preserving the image resolution. Fig. 5 shows the lateral profile through the center of the lesion at a channel SNR of $4.35 \mathrm{~dB}$. The edge of the cyst is sharper for the DAS + NLM algorithm. This illustrates that lesion detectability alone is not enough to ascertain the superiority of any imaging algorithm; as one can, trivially, trade off lesion detectability against spatial resolution. Lesion detectability should, therefore, be accompanied by an evaluation of the corresponding spatial resolution.

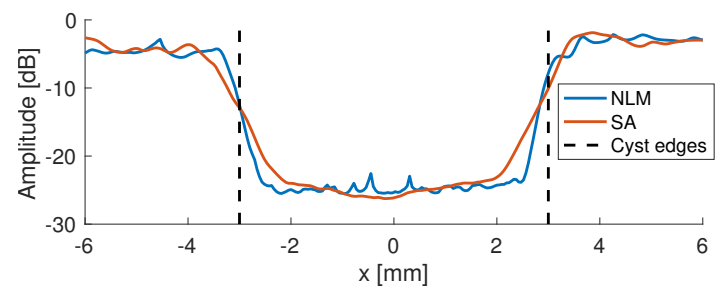

Fig. 5: Lateral profile through the center of the lesion for $\mathrm{SNR}=4.35 \mathrm{~dB}$ for DAS + NLM and DAS + SA. Lesion edges are indicated with dashed lines.

The results seem to indicate that coherence-based beamformers are not particularly well-suited for the task of detecting purely scattering lesions. Perhaps they can be better used to detect highly coherent targets, such as microcalcifications, or interfaces. Coherence beamforming has also been shown to be able to aid in the differentiation between solid and fluidfilled masses in-vivo [10].

The effect of the user settable parameters was briefly investigated. The SLSC implementation with a smaller kernel size $0.1 \lambda$, Fig. $6 \mathrm{u}$ to $6 \mathrm{x}$, led to lower GCNR values, Fig. $2 \mathrm{f}$, than those obtained with larger kernel size $\lambda$, Fig. $6 \mathrm{q}$ to $6 \mathrm{t}$ with the resulting GCNR in Fig. 2e. This may indicate that the GCNR improvement observed in SLSC may be due to this introduced spatial averaging.

Our results demonstrate that the entire signal processing chain in ultrasound imaging is responsible for the final performance. However, a full implementation of the entire chain is a demanding task for an individual researcher. Having an open source implementation of that chain, from channel data to post-processing of the image, such as the USTB (http://www.ustb.no), enable researchers to easily share their results and benefit from the others' implementations. We believe this can help increasing the quality and efficiency of the research done by the ultrasound community.

Lastly, it is important to point out that the implementations used here are not necessarily optimal for the detection of scattering targets. Other implementations, better tuned for the detection of particular lesion types, are of course possible. As an example, a real-time implementation of nonlocal-means in [11] demonstrated impressive results in despeckling of ultrasound images.

\section{CONCLUSION}

Post-processing of conventional delay-and-sum images using trivial spatial averaging and a edge-preserving nonlocal means, outperforms state of the art coherence beamforming methods at detecting scattering lesions. The methods' lesion detectability was evaluated using the Generalized Contrast-toNoise Ratio (GCNR), a metric that measures the maximum classification rate that can be achieved by an optimal observer. These results indicate that coherence-based beamformers are not particularly well-suited for the task of detecting purely scattering lesions.

\section{REFERENCES}

[1] O. M. H. Rindal, A. Austeng, A. Fatemi, and A. Rodriguez-Molares, "The Effect of Dynamic Range Alterations in the Estimation of Contrast," IEEE Transactions on Ultrasonics, Ferroelectrics, and Frequency Control, vol. 66, no. 7, pp. 1198-1208, 2019.

[2] A. Rodriguez-Molares, O. M. H. Rindal, J. D’hooge, S.-E. Måsøy, A. Austeng, and H. Torp, "The Generalized Contrast-to-Noise Ratio," IEEE International Ultrasonics Symposium, IUS, no. 6, pp. 1-4, 2018.

[3] J. A. Jensen and N. B. Svendsen, "Calculation of Pressure Fields from Arbitrarily Shaped, Apodized, and Excited Ultrasound Transducers," IEEE Transactions on Ultrasonics, Ferroelectrics and Frequency Control, vol. 39, no. 2, pp. 262-267, 1992.

[4] J. A. Jensen, "Field: A program for simulating ultrasound systems," Medical \& Biological Engineering \& Computing, vol. 34, pp. 351-353, 1996.

[5] A. Tristán-Vega, V. García-Pérez, S. Aja-Fernández, and C. F. Westin, "Efficient and robust nonlocal means denoising of MR data based on salient features matching," Computer Methods and Programs in Biomedicine, vol. 105, no. 2, pp. 131-144, 2012. [Online]. Available: http://dx.doi.org/10.1016/j.cmpb.2011.07.014

[6] J. Camacho, M. Parrilla, and C. Fritsch, "Phase coherence imaging," IEEE Transactions on Ultrasonics, Ferroelectrics, and Frequency Control, vol. 56, no. 5, pp. 958-974, 2009.

[7] P. C. Li and M. L. Li, "Adaptive imaging using the generalized coherence factor," IEEE Transactions on Ultrasonics, Ferroelectrics, and Frequency Control, vol. 50, no. 2, pp. 128-141, 2003.

[8] M. A. Lediju, G. E. Trahey, B. C. Byram, and J. J. Dahl, "Shortlag spatial coherence of backscattered echoes: Imaging characteristics," IEEE Transactions on Ultrasonics, Ferroelectrics, and Frequency Control, vol. 58, no. 7, pp. 1377-1388, 2011.

[9] A. Rodriguez-Molares, O. M. H. Rindal, O. Bernard, A. Nair, M. A. Lediju Bell, H. Liebgott, A. Austeng, and L. Løvstakken, "The UltraSound ToolBox," IEEE International Ultrasonics Symposium, IUS, pp. 1-4, 2017.

[10] A. Wiacek, O. M. H. Rindal, E. Falomo, K. Myers, K. FabregaFoster, S. Harvey, and M. A. Bell, "Robust Short-Lag Spatial Coherence Imaging of Breast Ultrasound Data: Initial Clinical Results," IEEE Transactions on Ultrasonics, Ferroelectrics, and Frequency Control, vol. 66, no. 3, pp. 527-540, 2019.

[11] L. H. Breivik, S. R. Snare, E. N. Steen, and A. H. Solberg, "Real-Time Nonlocal Means-Based Despeckling," IEEE Transactions on Ultrasonics, Ferroelectrics, and Frequency Control, vol. 64, no. 6, pp. 959-977, 2017. 

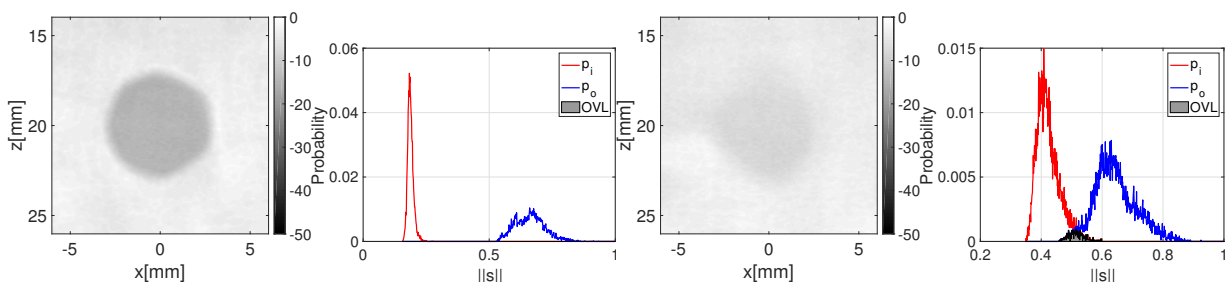

(a) $\mathrm{NLM}, \mathrm{SNR}=-4.85 \mathrm{~dB}$ (b) NLM, SNR=-4.85 dB (c) NLM, SNR=-14.07 dB(d) NLM, SNR=-14.07 dB
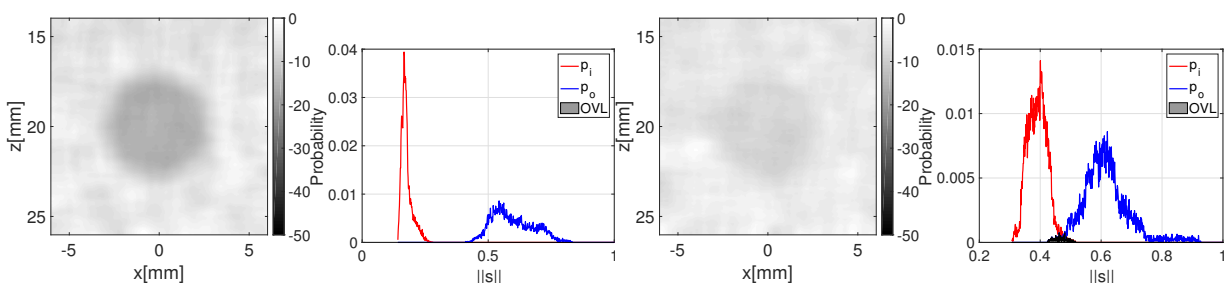

(e) $\mathrm{SA}, \mathrm{SNR}=-4.85 \mathrm{~dB}$

(f) $\mathrm{SA}, \mathrm{SNR}=-4.85 \mathrm{~dB}$

(g) SA, SNR=-14.07 dB

(h) SA, SNR=-14.07 dB
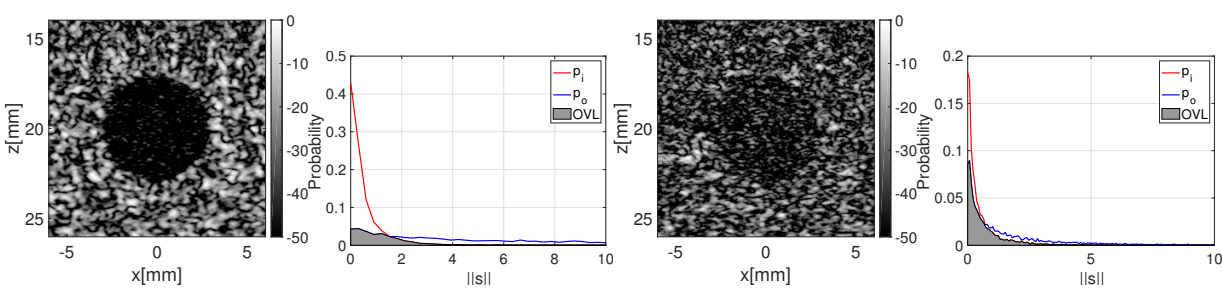

(i) $\mathrm{PCF}, \mathrm{SNR}=-4.85 \mathrm{~dB}$

(j) PCF, SNR=-4.85 dB

(k) PCF, SNR=-14.07 dB (l) PCF, SNR=-14.07 dB
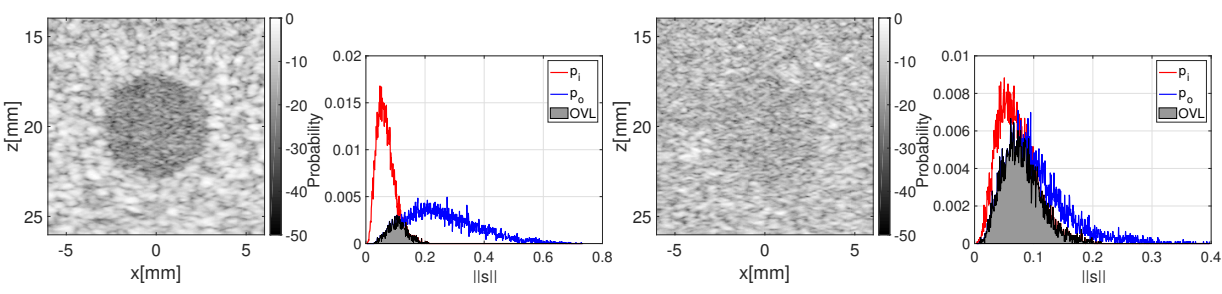

(m) GCF, SNR=-4.85 dB

(n) $\mathrm{GCF}, \mathrm{SNR}=-4.85 \mathrm{~dB}$

(o) GCF, SNR=-14.07 dB (p) GCF, SNR=-14.07 dB

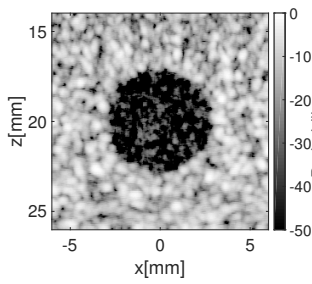

(q) $\operatorname{SLSC}(\lambda$ kernel) $\mathrm{SNR}=-4.85 \mathrm{~dB}$

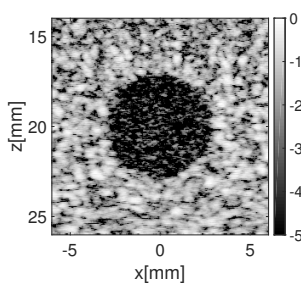

(u) SLSC (0.1 $\lambda$ kernel), $\mathrm{SNR}=-4.85 \mathrm{~dB}$

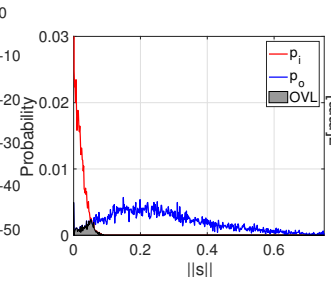

(r) SLSC ( $\lambda$ kernel), $\mathrm{SNR}=-4.85 \mathrm{~dB}$

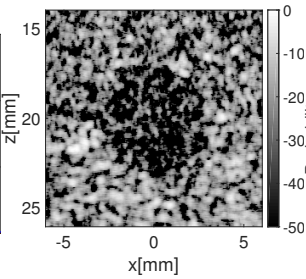

(s) SLSC ( $\lambda$ kernel), $\mathrm{SNR}=-14.07 \mathrm{~dB}$

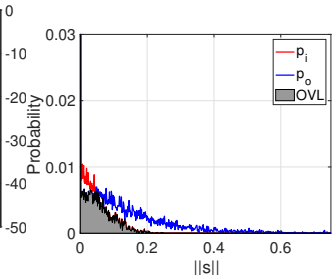

(t) $\operatorname{SLSC}(\lambda$ kernel), $\mathrm{SNR}=-14.07 \mathrm{~dB}$

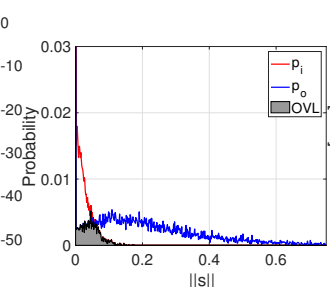

(v) SLSC (0.1 $\lambda$ kernel), $\mathrm{SNR}=-4.85 \mathrm{~dB}$

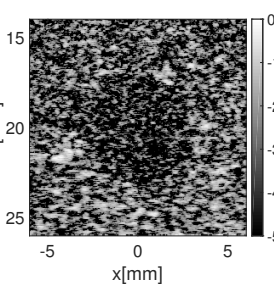

(w) SLSC $(0.1 \lambda$ kernel), $\mathrm{SNR}=-14.07 \mathrm{~dB}$

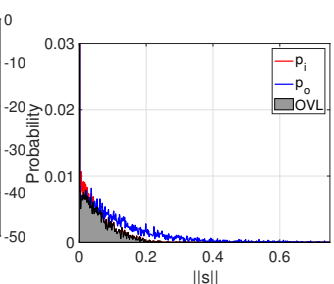

(x) SLSC (0.1 kernel), $\mathrm{SNR}=-14.07 \mathrm{~dB}$

Fig. 6: The firsth and third row is the b-mode images at respectively $-4.86 \mathrm{~dB}$ and $-14.07 \mathrm{~dB}$ SNR displayed at $-50 \mathrm{~dB}$ dynamic range. The second and forth row are the PDFs of the cyst and a region outside the cyst as highlighted in the DAS image in (b). In the plots of the PDFs the OVL region is indicated by the gray area. 